\section{MEANCALC: A BASIC program to calculate means and standard errors for a wide variety of designs}

\author{
MARK PLONSKY and ROBERT A. ROSELLINI \\ State University of New York at Albany, Albany, New York
}

In the course of research, it is often necessary for the investigator to calculate means and standard errors. One instance where such calculations are necessary is when knowledge conceming the progress of an ongoing experiment is desired. Another instance is when data are analyzed by statistical programs such as BMDP $2 \mathrm{~V}$ that do not provide means collapsed across one or more of the variables in the design. The present article describes a program that calculates means and standard errors quickly and easily for a wide variety of experimental designs and allows for the inspection of means collapsed across any selected variable or combination of variables.

There are basically four major advantages to the present program: (1) Cell means can be calculated with ease, and overall marginal means are also provided. A wide variety of designs can be accommodated, including those with several repeated measures. (2) Cell means can be collapsed over one or more of the independent variables in the analysis. As already noted, BMDP $2 \mathrm{~V}$ does not possess this capability, which can be a considerable aid in interpreting the results of an ANOVA. Although BMDP 8V does possess this capability, it is limited by the fact that all cells in the design must contain equal Ns. (3) Standard errors are computed. This capability is also lacking from the BMDP statistical package. (4) Finally, the program offers economical advantages. Typically, the cell means of an ANOVA are retrieved from the mainframe by using a terminal program such as HTERM (Kirk, 1980), and further analysis is performed via programs such as ANCOM (Shapiro \& Rosellini, 1980). With the present program, cell means can be computed from the raw data and saved to a disk file for future analysis, thus reducing the time (and its associated cost) of microcomputer-to-mainframe linkage. In addition, the need to run analyses before the experiment has been completed is reduced or, indeed, even eliminated by an examination of the output of this program.

Program Description. The program accepts up to 100 cases of data (with each case consisting of up to five data lines), three between-group variables, and three within-group variables. It allows for a total of 12 groups and up to 30 levels of repeated measures.

The authors' mailing address is: Department of Psychology, State University of New York, Albany, New York 12222.
Error-trapping routines do not permit these limits to be exceeded. The program requests the number and names of the factors (and levels) in the design as well as the number of cases. When calculations on raw data are made, each case is expected to consist of a group code followed by all of the data points in sequence. This data format is consistent with that used in the BMDP statistical package. Data files can be created with a text editor such as UNISIM (Shapiro, Rosellini, \& Decola, 1981) or with a word-processing program such as SCRIPSIT, as long as the files are saved in ASCII format. Since researchers often embed identifying information in the data file, an option that prepares a new file (with all identifying information removed) for use with the program is provided. This option is actually a second program that is invisible to the user.

The means-collapsing feature permits the user to obtain means collapsed over one or more of the independent variables in the analysis. It is noteworthy that this feature can be performed repeatedly, since the program stores the cell means and collapsed means in different areas of memory. If the user wishes only to collapse already existing cell means, an option is provided to input the cell means directly from disk.

Finally, the program output is in a format modeled after BMDP, with factor and level labels, as well as the Ns per group, displayed. The desired amount of rounding is requested, and the output can be sent to the screen or to a printer. Also, an option to store the cell means in a disk file is provided. See the Appendix for a brief sample run.

Program Requirements. These programs are written according to the syntax of Radio Shack Level II disk BASIC. The main program occupies approximately $10 \mathrm{~KB}$ of memory (the file-preparing utility occupies $3 \mathrm{~KB}$ and is compatible with either a $48-$ or $64-\mathrm{KB}$ system. These programs have been used successfully with TRSDOS, NEWDOS80, and DOSPLUS diskoperating systems.

Availability. A more detailed sample runstream, program listings, and copies of the programs on a minifloppy diskette can be obtained from the second author. To cover mailing, reproduction, and diskette costs, please include $\$ 10$.

\section{REFERENCES}

KIRK, R. C. HTERM/CIM and HTERM/BAS: "Smart" terminal enabling programs for the TRS-80 microcomputer. Behavior Research Methods \& Instrumentation, 1980, 12, 71-72.

Shapiro, N. R., \& Rosellini, R. A. ANCOM: A BASIC program for analytical comparisons among means. Behavior Research Methods \& Instrumentation, 1980, 12, 633.

Shapiro, N. R., Rosellini, R. A., \& Decola, J. P. UNISIM: A text-editing BASIC program for creation and modification of sequential disk files. Behavior Research Methods \& Instrumentation, 1981, 13, 689-690. 


\section{Appendix \\ Brief Sample Run}

The following example is taken from an experiment on animal learning. It is for the most part self-explanatory, and will be only briefly summarized here. It consists of a $2 \times 2 \times 3 \times 2$ mixedfactorial design. Treatment and schedule serve as the betweensubject factors, and block and day serve as the within-subject factors. The levels of treatment have been termed shock and control, and the levels of schedule are called VT10 and VT60. The total number of subjects (or cases) is nine. The following is a listing of the file containing the data. This file is called EXAMPLE/TXT.

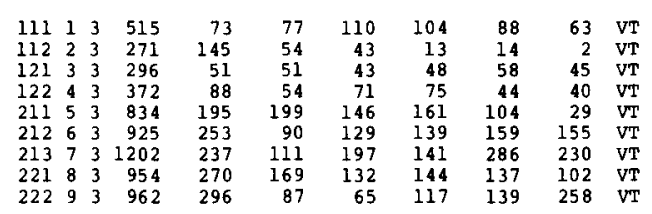

$\begin{array}{llllll}123456789 & 123456789 & 123456789 & 123456789 & 123456789 & 12\end{array}$

The bottom line contains a scale (and is not part of the data file). Columns 1 and 2 contain the group codes; columns 13 through 48 contain the data. The remaining columns contain identifying information, etc.; thus the file needs to be reformatted by the program before the means can be calculated.

In this example, cell means and standard errors are calculated, after which the cell means are displayed. Finally, means collapsed over the day variable are computed. Various features, such as the file-preparing option, could not be described due to space limitations.

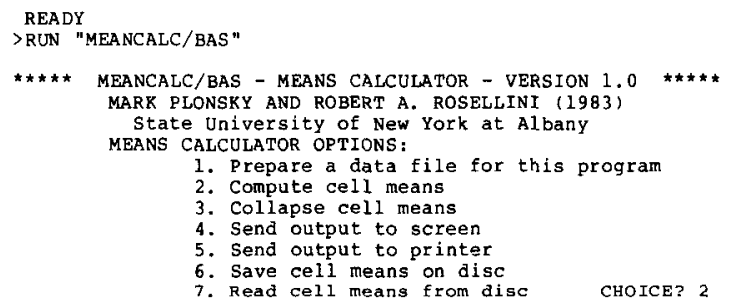

Number of between group I.V.'s? 2

Name (up to 6 characters) for BETWEEN group I.V. \# 1 ? TREATM Name (up to 6 characters) for BETWEEN group I.V. \& 2 ? SCHED Number of levels of TREATM ? 2

Number of levels of SCHED? 2

Name (up to 4 characters) for level 1 of TREATM? SHOC

Name (up to 4 characters) for level 2 of TREATM? CONT

Name (up to 4 characters) for level 1 of SCHED? VTlO

Name (up to 4 characters) for level 2 of SCHED? VT60

Number of within group I.V.'s? 2

ENTER A NAME FOR EACH, FROM THE FASTEST TO SLOWEST CHANGING.

Name (up to 2 characters) for WITHIN qroup I.V I? BL

Name (uP to 2 characters) for WITHIN group I.V. 2 ? DY

Number of levels of BL? 3

Number of levels of $D Y$ ? 2

FILE SPEC of the data $=>$ EXAMPLE/MCD: 1

Total number of cases?

Do you wish to divide all scores by a constant (Y/N)? N
The program then computes the means and standard errors, while providing numerous cues as to the calculations being performed. It returns with the following message-MEMORY CONTAINS THE FOLLOWING: Cell means and Standard errors for EXAMPLE/MCD:1, followed by the options table. Since we would now like to see some of the output, we choose Option 4: Send output to the screen. The type of output (cell means, standard errors, or collapsed means) is then requested, as is the format for this output. We choose the default, that is, accuracy to one decimal place. Note that the minimum and maximum means, as well as the range of means, are also presented (to aid in graphing the data).

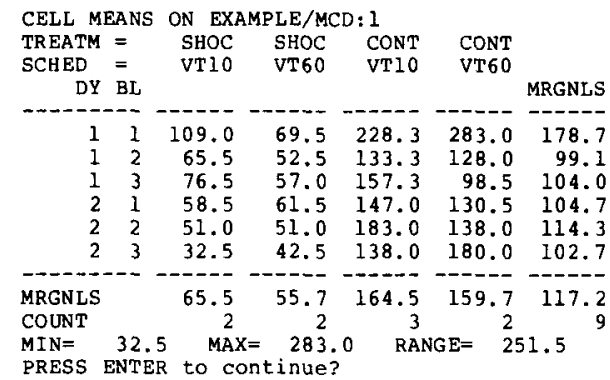

The options table is again presented, and this time we choose Option 3: Collapse cell means. Although in this example we collapse only over the day variable, note that any one or more of the variables could be collapsed. The following message is then presented:

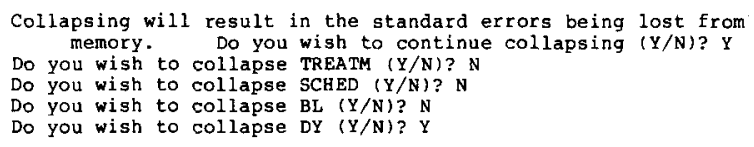

The program proceeds to collapse the means, which can then be displayed as follows:

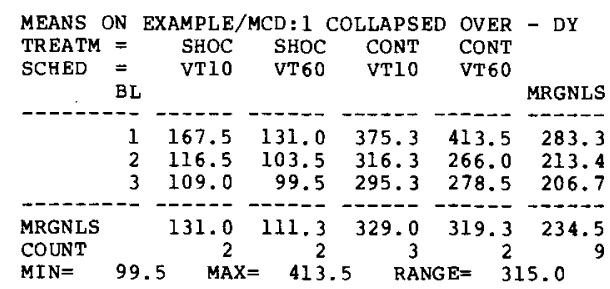

(Manuscript accepted for publication June 2, 1983.) 